\title{
A school based psychosocial intervention was effective in children with persistent post-disaster trauma symptoms
}

\author{
Chemtob CM, Nakashima JP, Hamada RS. Psychosocial intervention for postdisaster trauma symptoms in elementary \\ school children: a controlled community field study. Arch Pediatr Adolesc Med 2002 Mar;156:211-6. \\ QUESTION: In children with persistent disaster related trauma symptoms, what is the \\ effectiveness of a school based psychosocial intervention?
}

\section{Design}

Randomised (unclear allocation concealment*), blinded (outcome assessors)*, controlled trial with 1 year of follow up.

\section{Setting}

10 public elementary schools in Kauai, Hawaii, USA.

\section{Participants}

248 children in grades 2-6 (mean age 8 y, age range $6-12$ y, $61 \%$ girls) with persistent disaster related trauma symptoms 2 years after experiencing a major hurricane disaster. Follow up was $93 \%$.

\section{Intervention}

Children were allocated to 1 of 3 consecutively treated cohorts ( $\mathrm{n}=65,101$, and 82 in each cohort, respectively). Children in the cohorts awaiting treatment served as controls. Within each cohort, children were further allocated to receive individual $(\mathrm{n}=73)$ or group treatment $(\mathrm{n}=176)$ given by trained school based counsellors $(2$ men and 2 women). 4 weekly sessions of manual guided treatment focused on helping children master disaster related psychological challenges including restoring a sense of safety, grieving losses and renewing attachments, adaptively expressing disaster related anger, and achieving closure about the disaster to move forward.
Group and individual treatments used similar activities; however, children in group treatment also engaged in cooperative play and discussion.

\section{Main outcome measures}

Self reported trauma symptoms were assessed using the Kauai Recovery Inventory (frequency of reexperiencing, avoidance, and arousal symptoms in the previous week). Clinician rated post-traumatic stress disorder symptoms were assessed using the Child Reaction Inventory, but $<80 \%$ of children were assessed.

\section{Main results}

Children who were treated had fewer self reported trauma symptoms than those who served as controls (effect size $0.50, \mathrm{p}<0.001$ ); group and individual treatments did not differ in effectiveness. Individual treatment had a higher attrition rate than group treatment $(15 \% v 5 \%, \mathrm{p}<0.01)$.

\section{Conclusions}

In children with persistent disaster related trauma symptoms, a school based psychosocial intervention reduced disaster related trauma symptoms. Group and individual treatments did not differ in effectiveness but group treatment had higher retention rates.

*See glossary.
Source of funding: State of Hawaii.

For correspondence: Dr C M Chemtob, Mount Sinai School of Medicine, New York, NY,USA.

claude.chemtob@ med.va.gov

\section{COMMENTARY}

Reducing symptoms of trauma and conducting interventions on a large scale raise challenges nicely surmounted in this study by Chemtob et al. Children are exposed to all sorts of trauma including various national and natural disasters, crime, violence, abuse, and neglect. Screening and treatment that might be done on a large scale provide a useful first line intervention for helping children and families. This initial demonstration is an important step in identifying an intervention that might have broad applicability.

Comparing treatment with no treatment (waiting list) provides an excellent beginning. Critical questions urgently in need of attention focus on what ought to be accomplished next rather than limitations of this study. Two of the key questions pertain to the outcome. Firstly, does the intervention make any "real" (ie, genuine, palpable, and important) difference to the lives of the children? Statistically significant changes and effect sizes, quite important in their own right, are not necessarily connected to improvements in everyday life or adaptive functioning of the treatment group or individuals within that group. We need to know whether important changes were made and how many children showed these changes. Secondly, which children respond well or poorly to treatment? Two outcome measures were used. One of these was assessed in only 37 children. This would not permit identification of factors that may influence outcome and response to treatment.

Mounting clinical trials in schools and other naturalistic settings, as well as in clinical services, will require assessments that permit identification of outcome effects for all. It will be essential to know who and how many are likely to be helped by an intervention. Schools and communities that might wish to adopt such an intervention will want to know the costs (time, money, staff, and public relations with families) and benefits of treatment, and then make decisions on whether to adopt the intervention. Also, as a practical matter, parents will want to know when a treatment has not worked and what might be the next line of intervention. These investigators have identified an intervention clearly worth moving to these next steps.

Alan Kazdin, PhD, ABPP Yale University School of Medicine New Haven, Connecticut, USA 